\title{
Life Extending Control A Concept Paper
}

Carl F. Lorenzo and Walter C. Merrill Lewis Research Center

Cleveland, Ohio

$$
\begin{array}{llll}
\text { (NASA-TM-104391) LIFE EXTENOING CONTROL: A } & \text { N91-22135 } \\
\text { CONCEPT PAPER (NASA) } 17 P & \text { CSCL OIC } & \\
& & & \text { UnClas } \\
& & 63 / 08 & 0013493
\end{array}
$$

Prepared for the

American Control Conference

sponsored by the American Automatic Control Council

Boston, Massachusetts, June 26-28, 1991 
$\therefore \quad \therefore-\quad \cdots-=$ 
Life Extending Control

- A Concept Paper -

Cart F. Lorenzo and Walter C. Merrill

NASA Lewis Research Center

Cleveland, Ohio 44135

\begin{abstract}
The concept of Life Extending Control is defined. Life is defined in terms of mechanical fatigue life. A brief description is given of the current approach to life prediction using a local, cyclic, stress-strain approach for a critical system component. An alternative approach to life prediction based on a continuous functional relationship to component performance is proposed. Based on cyclic life prediction an approach to Life Extending Control, called the Life Management Approach is proposed. A second approach, also based on cyclic life prediction, called the Implicit Approach, is presented. Assuming the existence of the alternative functional life prediction approach, two additional concepts for Life Extending Control are presented. These are called the Measured Damage Approach, and the Estimated Damage Approach. A simple, hydraulic actuator driven, position control system example is used to illustrate the main ideas behind the Life Extending Control concept and is based on a cyclic fatigue damage model. Some results are given which demonstrate that system durability can be maximized by more effectively managing the resource of critical component life. Some additional research is proposed to develop a functional life prediction method and to demonstrate the concept of Life Extending Control.
\end{abstract}

\title{
INTRODUCTION
}

Systems with high performance requirements often have a small number of components that operate close to mechanical design margins. These critical components usually define the effective lifetime of the system and/or the mean time between maintenance of the system. Intelligent Control Systems (ICS) ${ }^{1,2}$, shown for example in Figure 1, address this issue at the time of incipient failure through the incorporation of active diagnostics and prognostics. This is followed by accommodation actions which are determined at a high level (Coordination Level) in the hierarchy and consider full mission requirements. The accommodation actions are implemented through the control system by: changing controller requests, and/or effecting controller structure or gain changes in the event of a large change in the plant dynamics. Typical of the objectives of such an Intelligent Control system are extension of system life and increased availability of the plant in the context of unknown disturbances or incipient hardware failure.

Another approach is to extend the lifetimes of critical components by the manner in which the system is controlled through transients. As part of the NASA Lewis Research Center program of Intelligent Control for Reusable Rocket Engines the concept of Life Extending Control (LEC) in now in development. This LEC concept proposes an active approach to managing the lifetime of the system critical components to extend the time between maintenance actions, or in the case of multiple mission objectives, to increase the total mission effectiveness of the system. This may be particularly true of systems which have long expected lifetimes, multiple mission objectives, and limited access to regular maintenance. Such characteristics are typical of long duration space missions. Life Extending Control in the Intelligent System context appears as an augmentation to the inner direct control loop shown in figure 1.

The objective of this paper is to describe several proposed approaches to the design of Life Extending Control(LEC) systems. This is accomplished by describing each concept and through the use of simple examples. In this paper the concept of LEC means the management of the resource of component lifetime and the achievement of a desired objective. The desired objective is usually defined in terms of control performance. The 
concept depends upon a prediction of the fatigue life of the particular component (or components) in the control system. This component is assumed to be the life limiting element, or most likely candidate for mechanical failure,

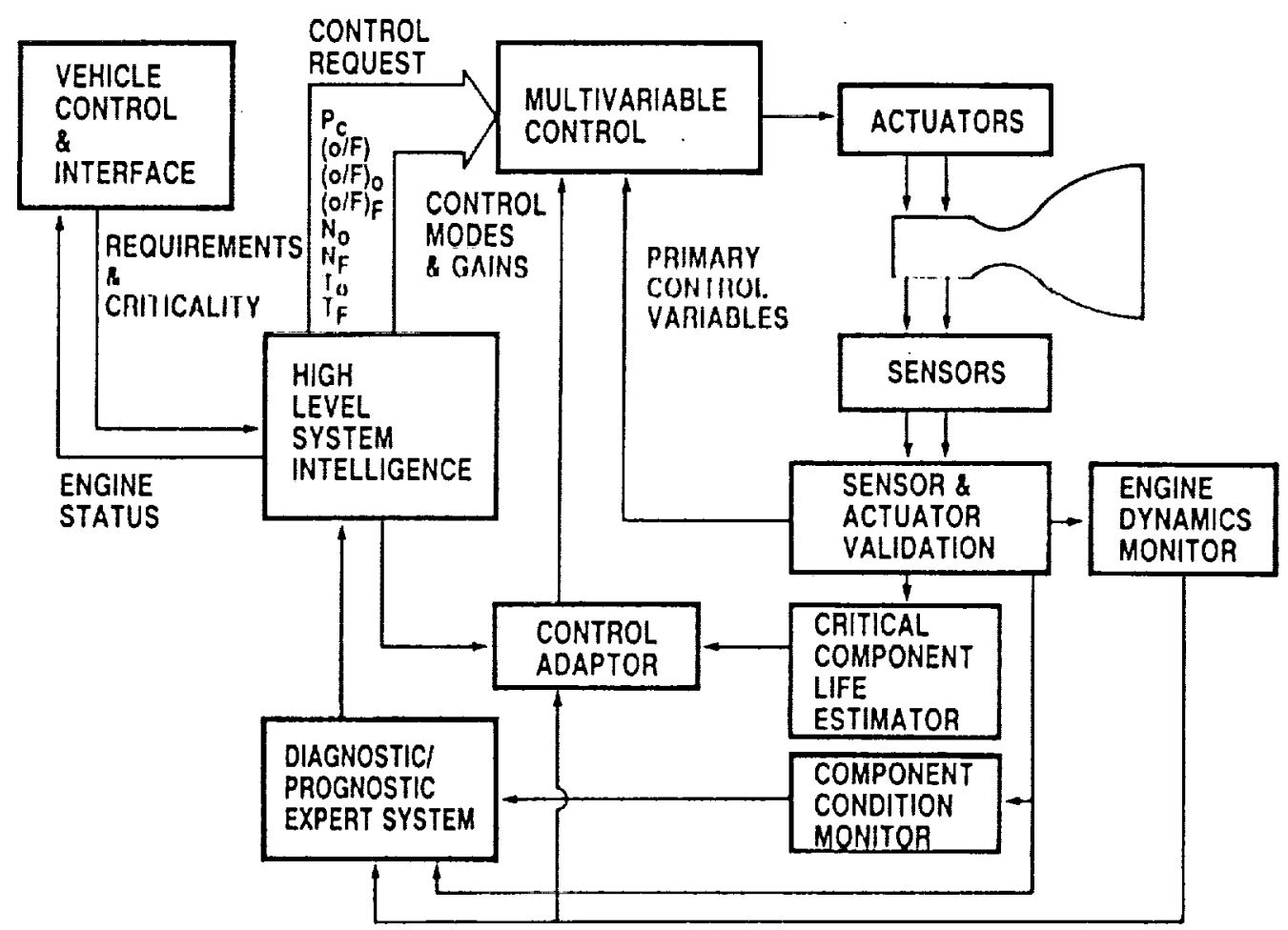

Figure 1 Reusable space propulsion intelligent control system framework

within the control system. Currently, the fatigue life prediction of this component is based on a local, cyclic strain approach described below. The prediction of the remaining life of the component as well as an understanding of the effects of cyclic loading, stress, and strain on remaining life enable a quantification of the tradeoff between system performance and life to be established for a dynamic system. Once this tradeoff is defined, a control which maximizes system performance for a given lifetime of the component (or conversely, which minimizes damage for a given system performance) can be designed.

The paper first describes the cyclic life prediction method and explains how it might be extended to a continuous functional life prediction approach. The life prediction procedure is analyzed to determine possible approaches to life extending control. Next, two approaches to LEC based on cyclic life prediction are presented. Then, two LEC concepts based on continuous function life prediction are presented. An example problem with a simple dynamic system and a fairly general performance measure is defined. Based on this example, some simulated results are presented which illustrate the main points and some of the possibilities of LEC. The paper concludes with some recommendations and possible approaches for future research in LEC.

\section{LIFE PREDICTION}

Both the current theory for life prediction and a proposed extension of this theory are presented.

\section{CURRENT LIFE PREDICTION APPROACH}

The cyclic life prediction process is highly nonlinear and comes from the study of metal fatigue due to cyclic loading. Usually the life estimates that result are used to forecast service life for a particular component 
design. Here that estimate will be calculated and used directly to manage life as the component undergoes transient loading during system operation. It is assumed to simplify the analysis that the life calculations can be based upon the stresses and strains estimated to occur locally at a single, critical point. This is a typical assumption used in life prediction analysis. The detailed procedure for life prediction is complicated and depends on many factors ${ }^{3}$. A simplified version of the procedure for life calculation is as follows. First the local strain, $\varepsilon$, is calculated from the load history, $F$, by

$$
\delta e=\frac{\delta F}{c_{1}}+\left(\frac{8 F}{c_{2}}\right)^{\frac{1}{d}}
$$

where $c_{1}, c_{2}$, and $d$ are constants that depend upon the material and

$$
\delta \varepsilon=\frac{\epsilon-\epsilon_{r}}{2} ; \quad \delta F=\frac{F-F_{r}}{2}
$$

with the subscript $r$ indicating a reference point. The determination of the reference point is critical to the calculation procedure and involves a careful ordering of the hysteresis like stress-strain cycles by magnitude and direction. These cycles are described by

$$
8 e=\frac{80}{E}+\left(\frac{80}{A}\right)^{\frac{1}{s}}
$$

where $\sigma$ is the stress, $\delta \sigma=\left(\sigma-\sigma_{\mathrm{r}}\right) / 2$, and $\mathrm{E}, \mathrm{A}$, and $s$ are material constants. Once the stress-strain cycles are calculated, the life usage associated with a single cycle of the given load history can be estimated from

$$
\delta_{\varepsilon}=\left(\frac{\sigma_{f}-\sigma_{\text {avo }}}{E}\right)\left(\frac{2}{D_{l}}\right)^{b}+e_{f}\left(\frac{2}{D_{l}}\right)^{c}
$$

Here $\sigma_{1}, E, b, \varepsilon_{1}$, and $c$ are material constants, $D_{1}$ is the life lost due to cycle $i$, hereafter called damage, and $\sigma_{\text {ave }}$ is an average tensile stress which is used to correct for nonzero mean tensile stresses in predominately elastic cycles. Once the cyclic analysis has been completed, total damage may be estimated by

$$
D=\sum_{l} D_{l}
$$

using the Palmgren-Miner approach ${ }^{3}$. Other, more accurate and complex approaches to determining total damage given the cyclic analysis are possible ${ }^{4}$.

The procedure of equations (1)-(4) can be solved at discrete instances of time, l.e. whenever a stressstrain cycle has been completed, elther by a traditional computing approach or by a neural network. This dependence on cycle closure for updating the effect of loading on component life makes a direct application of the current life estimation procedure to life extending control impossible. An indirect application is possible and two indirect approaches will be described in a later section. However, the direct application of a life estimation procedure to control would be more beneficial and is therefore the ultimate goal of life extending control. To accomplish this goal, a new formulation of the life estimation procedure is required. This new formulation would consist of a continuous form of the damage laws instead of the current forms which require bookkeeping the number of cycles, their respective amplitudes, and their order of occurrence.

\section{CONTINUOUS LIFE PREDICTION APPROACH}

To achieve a continuous formulation of the life prediction process, an interdisciplinary approach is required. Here the knowledge of material properties and life prediction of fracture and fatigue scientists must be combined with the control engineers' knowledge of dynamics and modeling to develop these continuous forms. The 
objective would be to functionally relate measurable performance information with a differential form of the damage laws. This would allow the direct use of the differential estimate of damage in the life extending control law and when integrated over complete cycles, would give equivalent or superior damage predictions to those associated with the cyclic theory. Two approaches are possible here: 1) derive such forms from basic theory (the current research thrust of the field) or 2) empirically select likely forms with a significant number of unspecified parameters and use optimlzation theory to best "fit" the parameters using available data sets.

Adopting the second approach, several elementary forms, given in table I, are proposed. These forms are determined either by intuition or by observing the important relationships embodied in the current theory of life prediction. Various weighted, linear and nonlinear combinations of these elementary forms would be linearly regressed against the available fatigue life data to obtain a continuous formulation. Alternately, elementary forms consisting of the performance variables of the system, shown in table II, could be used. The difficulty with using the performance variables directly is that the critical stresses and strains are less easily addressed and the stresses and strains are nonlinear functions of the performance variables. In either case the combined form will generate a continuous estimate of component damage which can be used directly in a control procedure to minimize accumulated damage.

$$
\begin{aligned}
& \mid \text { Mean Stress Level }|=| \frac{1}{T} \int_{0}^{T} \sigma(t) d t \mid \\
& \mid \text { Mean Strain Level }|=| \frac{1}{T} \int_{0}^{T} e(t) d t \mid \\
& \mid \text { Cyclic Stress } \mid=\int_{0}^{T}\left(\sigma^{2}-\left[\frac{1}{T} \int_{0}^{T} \sigma(t) d t\right]^{2}\right) d t \\
& \mid \text { Cyclic Strain } \mid=\int_{0}^{T}\left(\epsilon^{2}-\left[\frac{1}{T} \int_{0}^{T} e(t) d t\right]^{2}\right) d t \\
& \left|\sigma T \eta_{;}\right| \epsilon T|;| \sigma e T \mid \\
& \oint_{C} \sigma d \varepsilon ; \oint_{C} \sigma T d E \\
& (a \sigma+b) \oint_{C} \sigma d e \quad \text { otc. }
\end{aligned}
$$

Table I. Elementary damage prediction forms

\section{LIFE EXTENDING CONTROL}

The fundamental concept of life extending control is to control rates of change and levels of some performance variables to minimize damage (or damage rates) critical components while simultaneously maximizing dynamic performance of the plant. It is emphasized that a fundamental tradeoff exists between the level of achievable performance and the ability to extend the life of system components generating that performance.

Since the life prediction procedure is grossly nonlinear and is intimately connected with the determination of stress-strain hysteresis cycles, any control strategy will depend upon the total time history of the force applied to the critical component. However, as long as some flexibility in obtaining system transient performance exists, the opportunity to manage life usage exists. A study of the life prediction procedure Indicates two factors which will be important when devising a control strategy, strain cycle magnitude and average tensile stress.

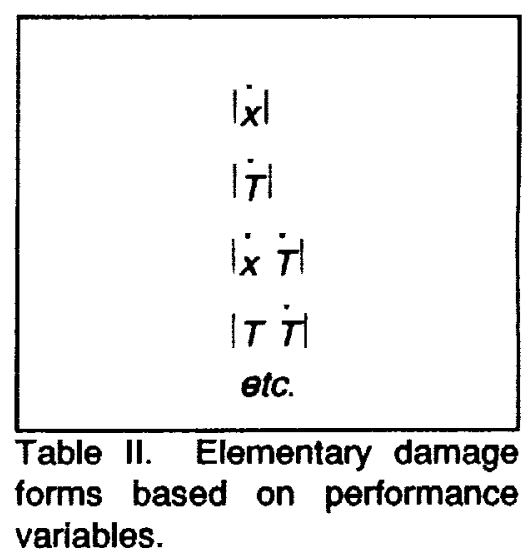

From flgure 2, which is a solution of equation (4), it is clear that life usage is directly related to strain magnitude. Strain magnitude is directly related to force. Thus, a control which minimizes forces applied to the critical component will extend life. This is hardly remarkable. However, LEC allows a comparison of two different control objectives based upon a quantitative analysis of the forces required to accomplish the different objectives. As assumed in this work, the time to achieve control performance can be varied to manage the forces and therefore the life of the critical component. In this way total system performance over the life of the component can be maximized.

Also, from flgure 2, it can be seen that nonzero average tensile stress applied during predominately elastic load cycles can substantially degrade life. For example if a particular commanded trajectory has left the component with a nonzero average tensile stress, small force perturbations(due perhaps to noise) will be more damaging than if they occur after a commanded trajectory that results in a average compressive stress. This 
shows clearly the need to plan the commanded trajectory over its time history.

A third factor, not explicitly shown by the equations, is component temperature. Higher component temperatures can have a marked effect on life usage. Higher temperatures change the material property constants in equations (1)-(4) and increase the life usage for comparable values of strain. These effects, although important, will not be considered in this paper. The basic ideas of life extending control can be more effectively illustrated without the consideration of this additlonal factor.

Four general approaches to LEC are presented. The first two are based on the cyclic life prediction approach and are called the Implicit Approach and the Life Management Approach. Assuming the existence of the alternative functional life prediction approach, two additional concepts for Life Extending Control are presented. These are called the Measured Damage Approach and the Estimated Damage Approach.

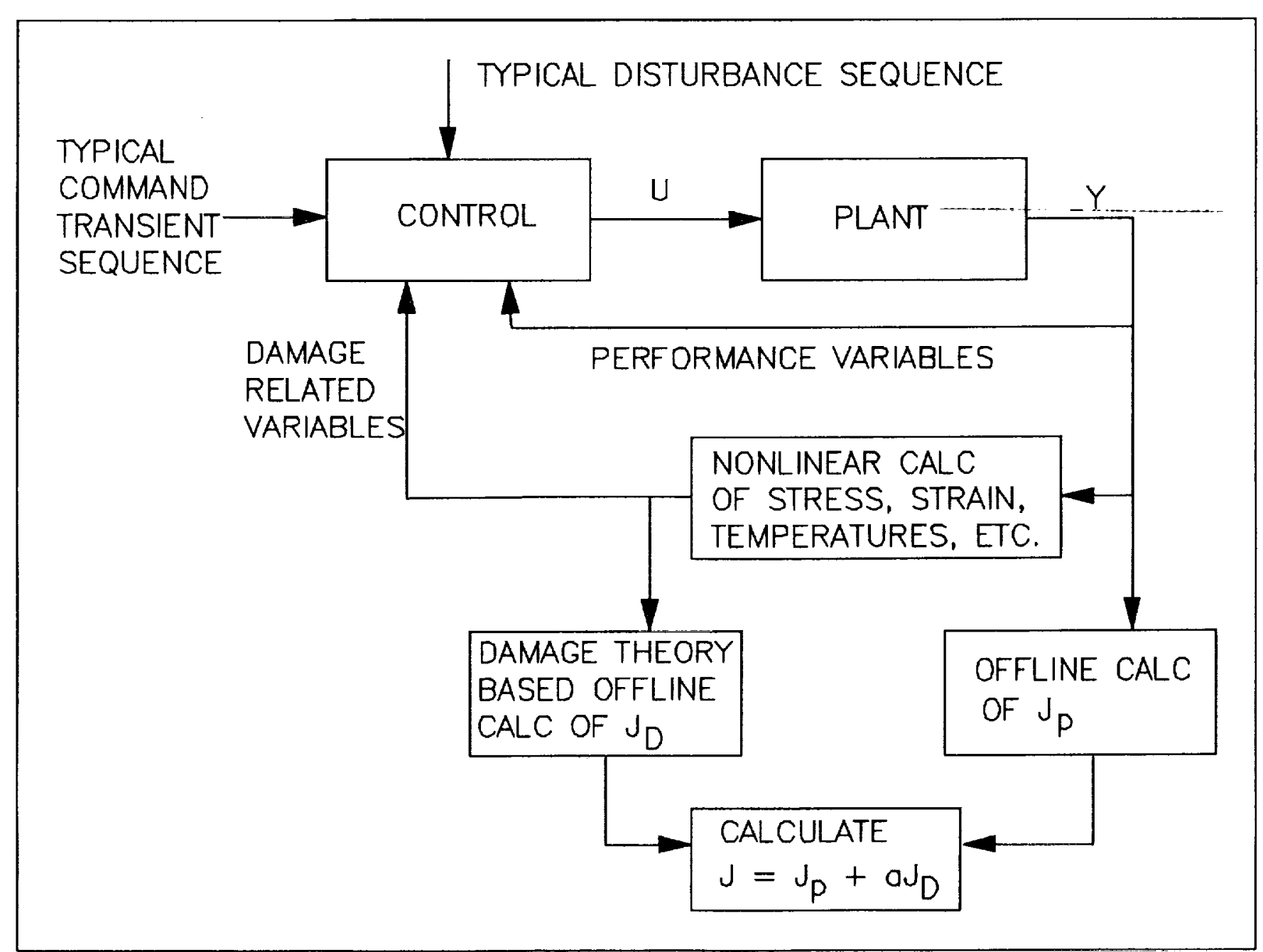

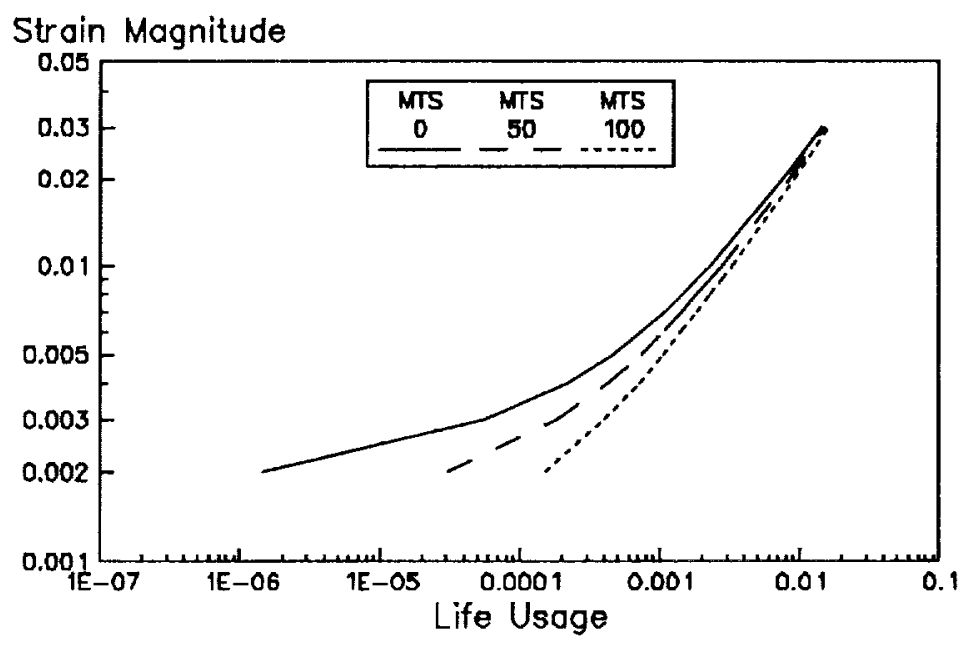

Flgure 2. Life usage versus component strain parameterized by mean tensile stress.

Figure 3. Implicit life extending control approach 
The implicit approach to Life Extending Control recognizes that current fracture/fatigue science cannot predict the differential damage on less than a full cycle of strain. The implicit approach (see figure 3) selects a sequence of typical command transients (and disturbances) that are representative of those the system would experience in service. Two performance measures are defined: $J_{p}$, an objective function that maximizes dynamic performance (possibly by minimizing quadratic state and control excursions) and $J_{0}$ a damage measure which uses the best (current) fatigue/fracture theory available to calculate the damage accumulated over the sequence of command transients. An overall performance measure can also be defined as

$$
J=J_{p}+a J_{D}
$$

where a represents the relative importance between performance and life extension. The implicit approach then selects a "best" control algorithm which is applied for the full sequence of command transients. The dynamic performance and damage accumulation over the sequence are optimized (relative to the selected measures) against the control algorithm parameters. The expectation is to find an algorithm such that the loss in dynamic performance is small (i.e. $J_{p, z, m m}-J_{p, o, m m}$ in flgure 4), for a significant reduction in accumulated damage over the sequence of transients (i.e., $\mathrm{J}_{\mathrm{D}, \mathrm{o}, \mathrm{min}}$ $J_{D, z, \text { min }}$ is large and life is extended). Here the subscript o refers to optimizing for dynamic performance only. An actual operating gain set (point $q$ in flgure 4) is then chosen which satisties the desired weighing between performance and damage (i.e. J). The

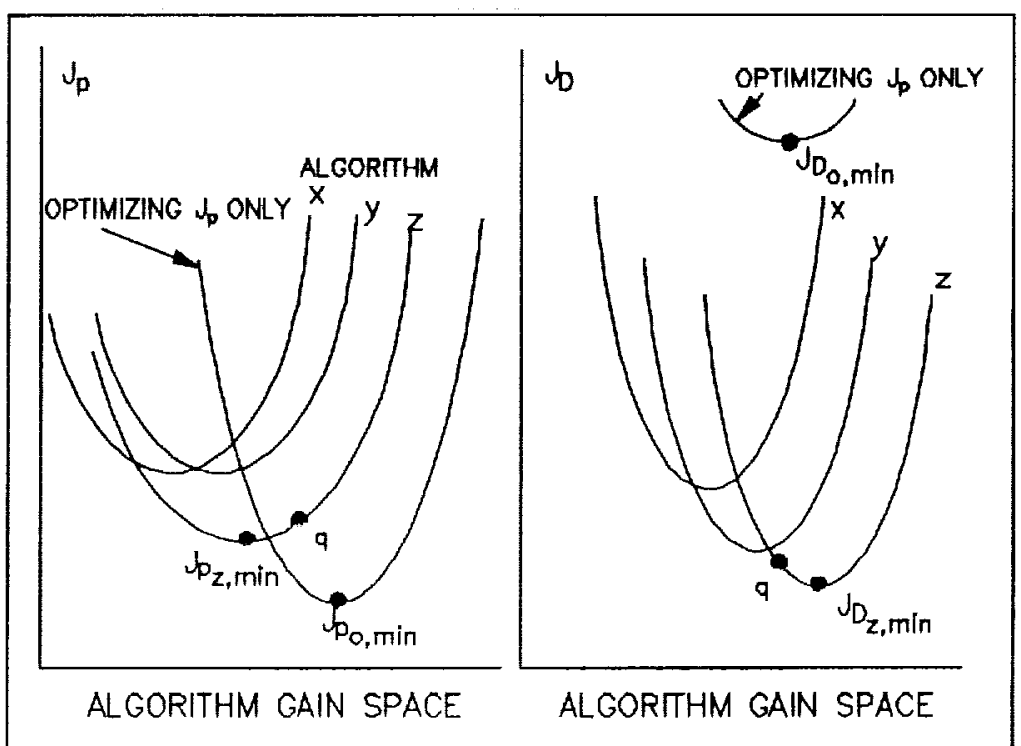

Figure 4. Effect of various life extending control algorithms on performance $\left(J_{p}\right)$ and damage $\left(J_{D}\right)$.

mechanics of the implicit approach are detailed as follows. During the design process, two types of feedback variables are considered: 1) the performance variables normally used to manage dynamic performance and 2)nonlinear functions of the performance variables representative of the damage variables (stresses, strains, temperature and various rates). Various control algorithms are then examined within this feedback structure. That is, the sequence of selected performance and disturbance transients are applied to a simulated system with a trial control and performance $J$ (or $J_{p}$ and $J_{D}$ separately) is calculated. Superior LEC algorithms can then be identifled as those that minimize $J$ (or $J_{p}$ and $J_{D}$ separately). A family of algorithms can be developed which are parameterized by the relative tradeoff parameter a. The final control can be selected from this set of algorithms with confidence that an effective control and a desirable performance/life tradeoff have been established.

At first to determine suitable LEC algorithms, the incremental damage law assumption is not made. However, intuitive LEC algorithms are readily formulated. Clearly minimizing the mean tensile stress, mean strain, and temperature levels and minimizing the cyclic amplitude of stress, strain, and temperature should minimize damage. Also, minimizing the number of cycles of stress and strain should contribute to extending critical component life.

For a typical application the elementary forms presented in table I singlely or in combinations would be tested for effectiveness as described above. Also, the performance variable forms of table II could be used directly. The problem with using the performance variables directly is that the critical stresses and strains are nonlinear functions of the performance variables. These nonlinear functions may be quite complicated and the essential information about damage accumulation may be lost. 


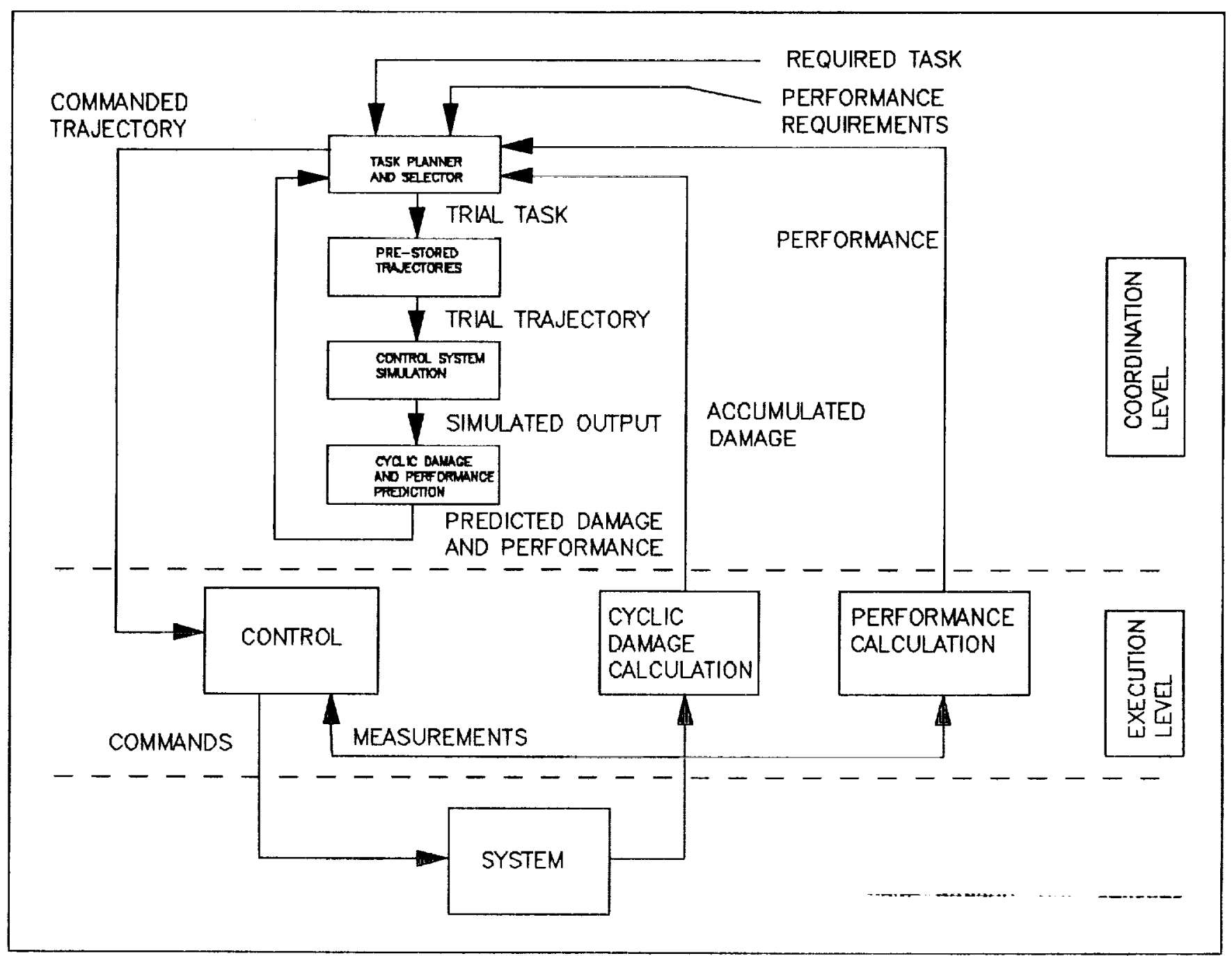

Figure 5. Life management life extending control approach.

\section{LIFE MANAGEMENT LEC}

The second indirect approach is referred to as the life management approach and is given in block diagram form in flgure 5. Here the LEC would have a hierarchical structure similar to that found in other proposed intelligent control systems (ICS) ${ }^{t}$. Here we define an ICS to be a control system that has coordination and execution levels and is based upon the principle of increasing precision with decreasing intelligence ${ }^{6}$. At the coordination level, the task planner uses performance requirements and balances these against life usage and appropriate control commands or strategies are selected. This is accomplished by simulation of the system for a few pre-selected trajectorles. From the results of this simulation and for a given performance definition, a commanded trajectory is selected that optimizes system performance and minimizes component damage over a sub-interval of the task. Within the task planner, information from a cyclic damage prediction model ranks various candidate trajectories for the successive interval in the planning and selection process. Outside of the task planner another cyclic prediction model assesses the actual damage accumulated during a sub-interval. The execution level implements the selected strategy in the Interval by translating the commanded trajectory into control commands and applying these commands to the system.

\section{CONTINUOUS LIFE PREDICTION BASED CONTROL}

The control approaches taken above do not directly control the damage rates of critical components. Direct control will require a continuous form of the damage laws instead of the current cyclic forms. In the sections which follow the existence of such a form is assumed. 


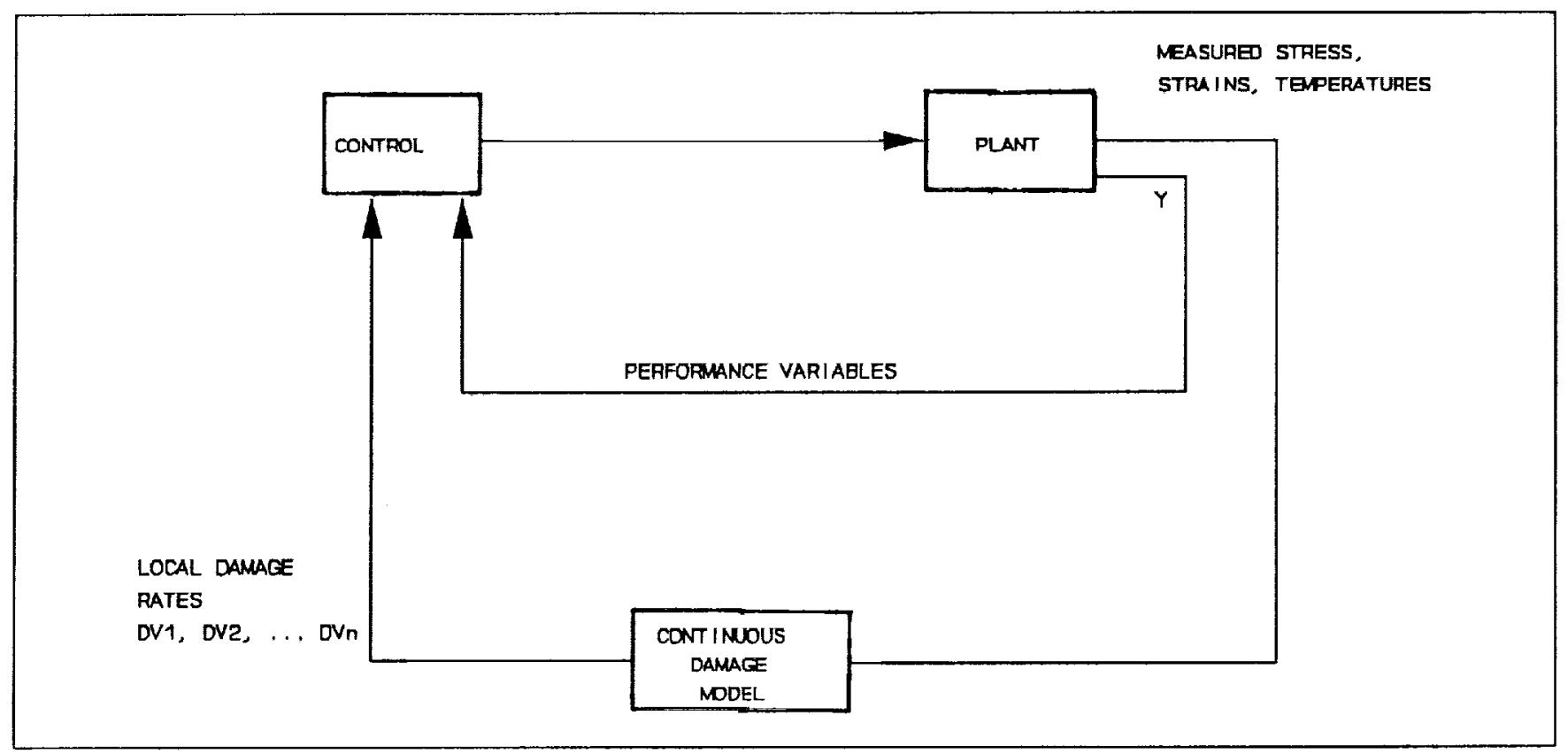

FIgure 6 Measured damage variables life extending control approach.

\section{DIRECT LIFE EXTENDING CONTROL}

In this approach (figure 6) both the plant performance and the damage (measured stresses, strains, temperatures, forces, etc.) related variables assoclated with critical components are measured and used as feedback information for the control. Here the control attempts to directly regulate life as a resource rather than indirectly as in the previous approaches. It is presumed that a "real-time" predictive damage (or life) model (described above) exists that would allow the prediction of the incremental damage (or local Damage rates DV1, DV2, ...) as a continuous function of measured stressses, strains and temperatures. Also, the influence of changes in the performance variables (presumed to be controllable) on the behavior of the critical life varlables is known. Thus, in figure 7, at time A, the damage associated with damage variable DV1 can be predicted for any control action (here actions $u_{1}, u_{2}$, and $u_{3}$ are considered and result in damages $D_{1}, D_{2}$, and $D_{3}$, respectively). (Note, damage while shown as a continuous function of time, will likely be modelled as a continuous function of local stress, strain, etc.) The control problem then is to minimize damage of the critical life components while maximizing (dynamic) performance of the plant. The performance objective approach of equation (6) can be used to achieve this optimization.

One implementation of a measured damage variables LEC would achieve control performance by adaptively modifying the control feedback structure to permit damage to accumulate at a "setpoint" rate, a linear rate over time for example. The measured damage variables could be used directly in a feedback law or to modify the gains or even the structure of the existing control. The emphasis here is on obtaining desired system operation by an active, feedback control approach. The issue of controllability is fundamental to such an approach.

\section{ESTIMATED DAMAGE VARIABLES LEC}

Unlike the Measured Damage Variables LEC approach, this concept, shown in flgure 8 uses a real time model to estimate the damage rates (and damage accumulation) of critical components. The models can be

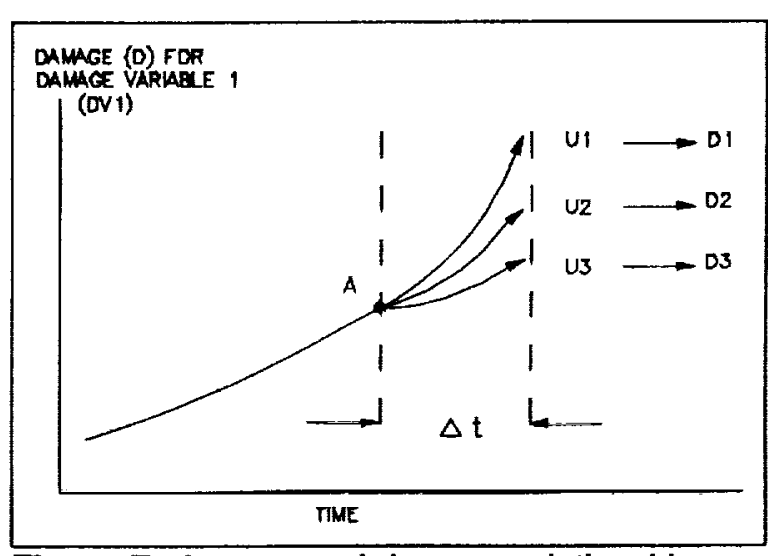

Figure 7. Incremental damage relationship 
driven by performance variables or performance variables augmented by available damage measures. Conceptually the models can vary from simple, precomputed, linear, influence coefficients to detailed, non-linear, real time structural models which may require considerable computation. These models would be a direct consequence of the continuous life model described above and would result in a damage estimator that estimates real time damage rates based on performance variables and/or structural dynamic model. The controller design would follow in much the same manner as for the measured damage variables approach.

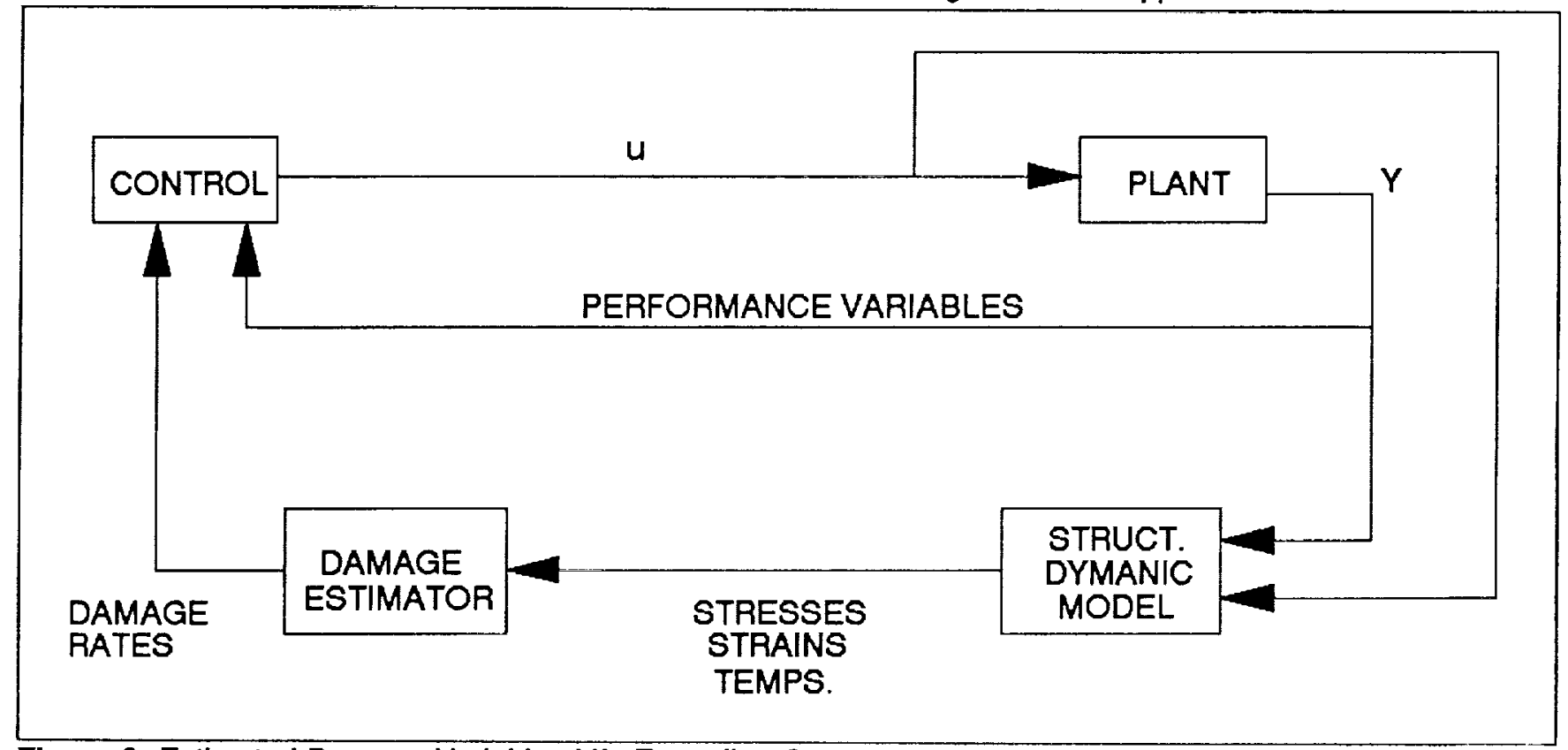

Figure 8 Estimated Damage Varlables Life Extending Control Approach.

\section{ESTIMATED DAMAGE LEC EXAMPLE}

As an example of Estimated Damage LEC, an advanced, reusable rocket propulsion system hierarchical control ${ }^{7}$, as shown in Figure 9, is studied. The propulsion system control hierarchy consists of two levels, the propulsion control level and the engine control level. The propulsion system consist of three separate, nominally identical, engines which need to be coordinated to give the desired thrust as determined by mission requirements. This coordination is accomplished at the propulsion control level. As shown in Figure 9, information on the thrust and the current life status of the engine are transmitted from the engine level to the propulsion level. Each of the engines has its own, independent engine control which exlsts at the engine control level.

Each engine in the propulsion system consists of a fuel turbopump, an oxidizer turbopump, and a main combustion chamber. The turbopumps consist of a preburner, turbine, and pump. Fuel and oxidizer are burned in the preburners. The hot gas generated in the preburners drive the turbines which in turn drive the pumps. The temperature of the gas discharged from the turbine is an important indicator of the relative health of the engine system. The control of the engine is accomplished by controlling by three valves, which adjust 1) the amount of oxidizer in the fuel turbopump, 2) the amount of oxidizer in the oxidizer turbopump, and 3) the relative split of fuel flow between the fuel turbopump and the oxidizer turbine.

As mentioned above, the fuel and oxidizer turbine discharge temperatures, $T_{F}$ and $T_{0}$, respectively, represent important indicators of engine health. A simplified life model using these indicators to illustrate the ideas of the Estimated Damage LEC approach is used in this example. Here relative damage rate, $D_{i}$, for the engine is empirically approximated as:

$$
D_{1}=k_{11}+k_{21} \operatorname{Max}\left(T_{F_{1}}-T_{F N_{1}} T_{O_{1}}-T_{O N_{1}}, 0\right)+k_{3 i} F_{1}^{2}
$$

where the k's are constants, $T_{F N}$ and $T_{O N}$ are the fuel and oxidizer turbopump discharge nominal temperatures, 


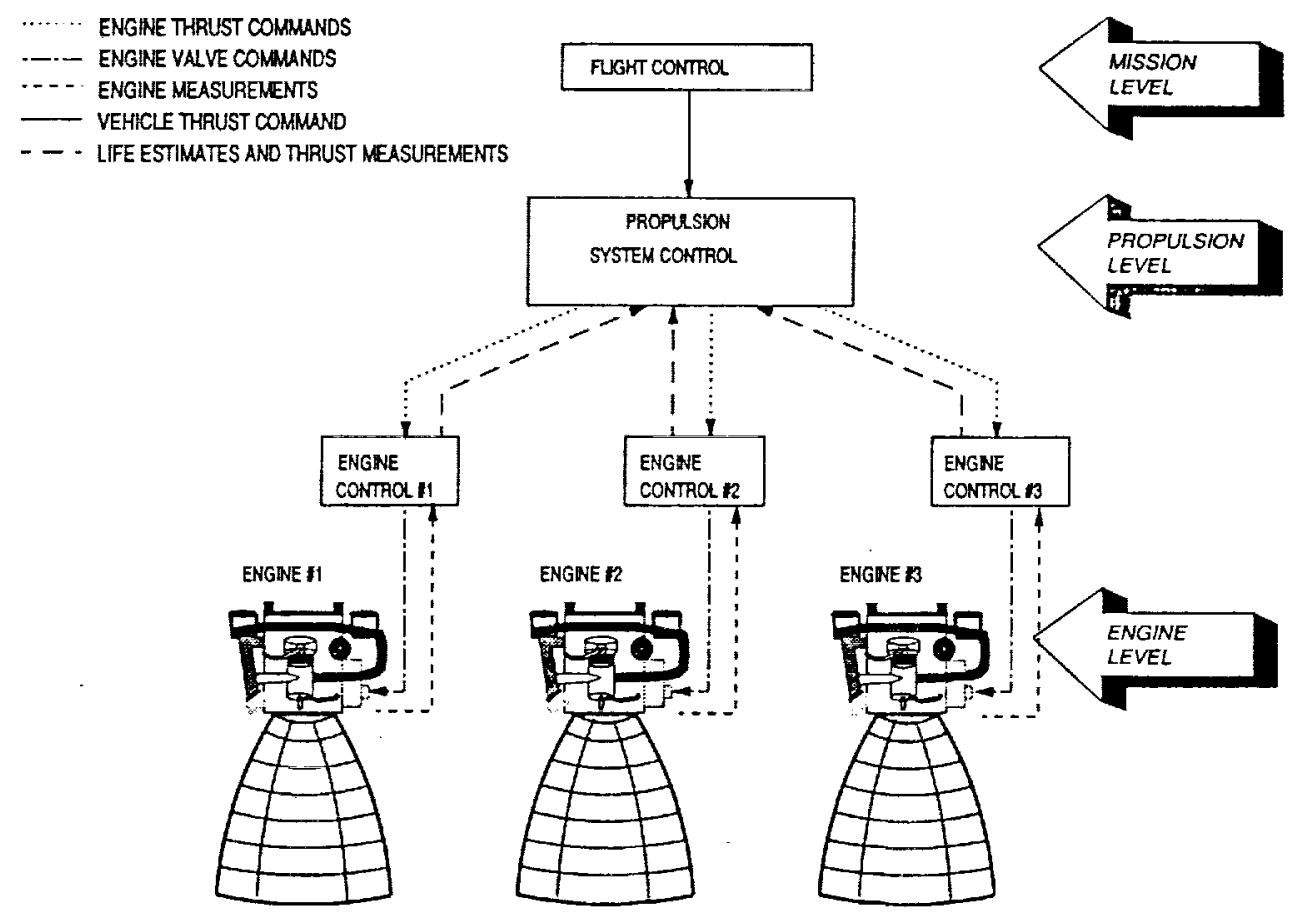

Figure 9 Hierarchical propulsion control system

respectively, and $F$ is thrust. In a gross sense, if either of these temperatures rise above a nominal level, the engine has degraded and has less useable life remaining. The nominal discharge temperatures would be a function of the power level of the engine. Thus, if the engine runs "hot" at any power level, e.g. $T_{F}-T_{F N}>0$, damage will accumulate faster than the nominal damage rate.

The control structure for the propulsion level control is shown in Figure 10. The propulsion level control has two goals, 1) control individual engine thrusts to the vehicle thrust command, and 2) force the individual engine damage rates to be as nearly equal as possible. Here the thrust from each engine is combined to give a vehicle thrust measurement for comparison with the desired vehicle thrust. Also, the damage rates are combined linearly to automatically adjust individual thrust commands to the engine level controls to achieve equal damage rate for each engine. Example results are given in Figure 11. Here, there is a requested increase in vehicle thrust at $t=0$ sec. to which the propulsion system responds. Additionally, it is assumed that engine \#3 degrades linearly throughout the example starting at $t=t_{1}$ sec such that $T_{F}-T_{F N}=k\left(t-t_{1}\right)>0$, where $k$ is a constant. Between $t_{1}$ and $t_{2}$, the control achleves equal damage rates for each engine while malntaining the vehicle thrust level. This is achieved by a downthrust of engine \#3 and an upthrust of engines \#1 and \#2. At $t=t_{2}$, engines \#1 and \#2 are at their maximum thrust levels and the degradation of engine \#3 can no longer be mitigated without reducing the vehicle thrust level. Therefore, between $t_{2}$ and $t_{3}$ engine $\# 1$ and $\# 2$ thrust levels remain constant and the \#3 damage rate increases beyond the \#1 and $\# 2$ levels. At $t_{3}$ the mission commanded vehicle thrust is reduced and the propulsion level implements the reduction using only engine $\# 3$, the engine displaying the highest level of life usage, until $t_{4}$ where the damage rates are again equal. Between $t_{4}$ and $t_{5}$, the decreasing vehicle thrust command is implemented by thrust reductions in all three engines that result in equal damage rates. At $t_{5}$ engine \#3 is at its minimum thrust and can no longer be downthrust. Since the engine continues to degrade, and is fixed at the minimum thrust level, the damage rate rises until the end of the simulation. At all times and for each event in the simulation scenario, the propulsion level coordinator commands the individual engine thrust levels to values that minimized engine wear rate and risk, as defined by the damage rate model, within the mission level and engine operation constraints. 


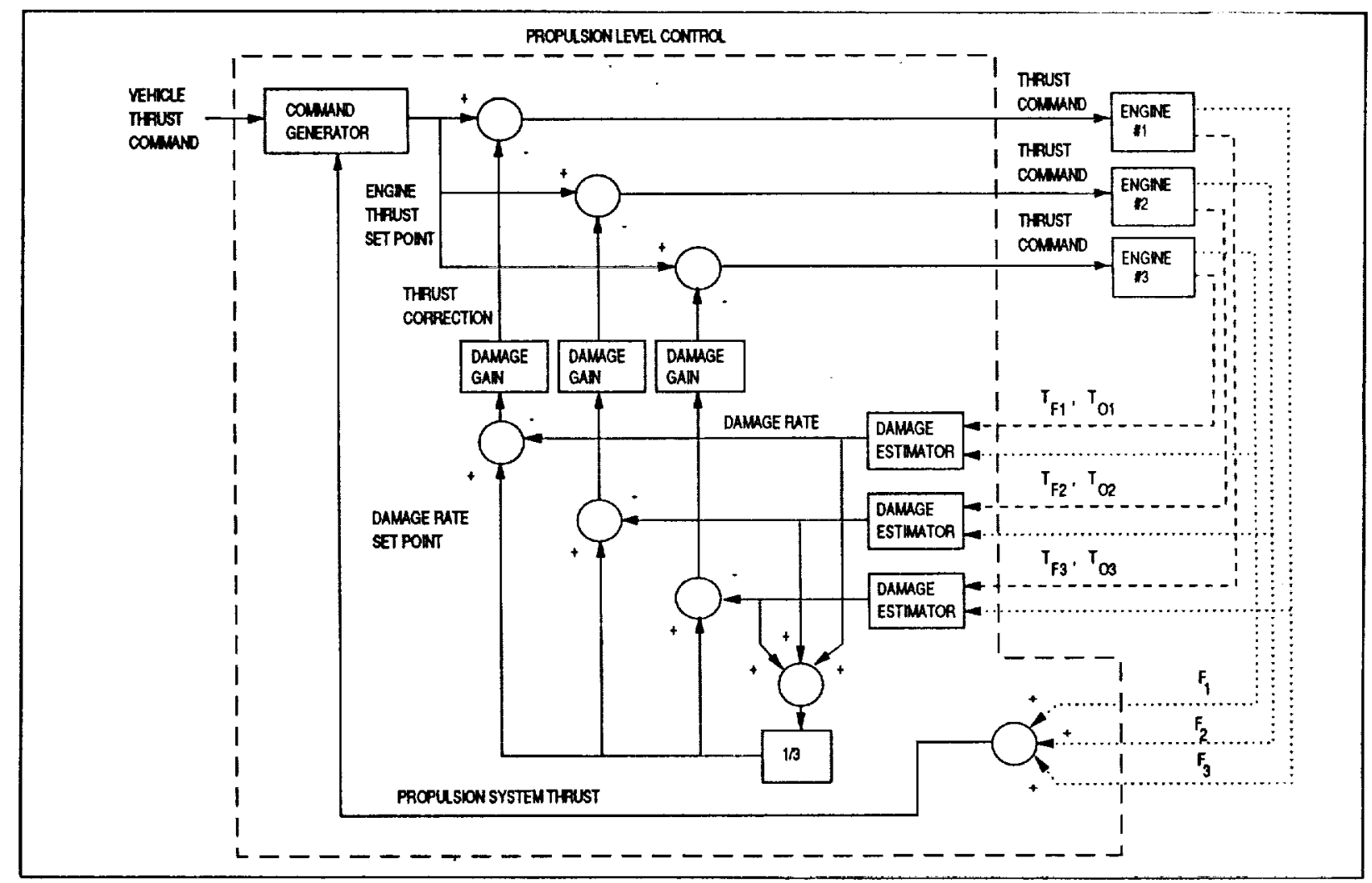

Figure 10 Propulsion level control structure

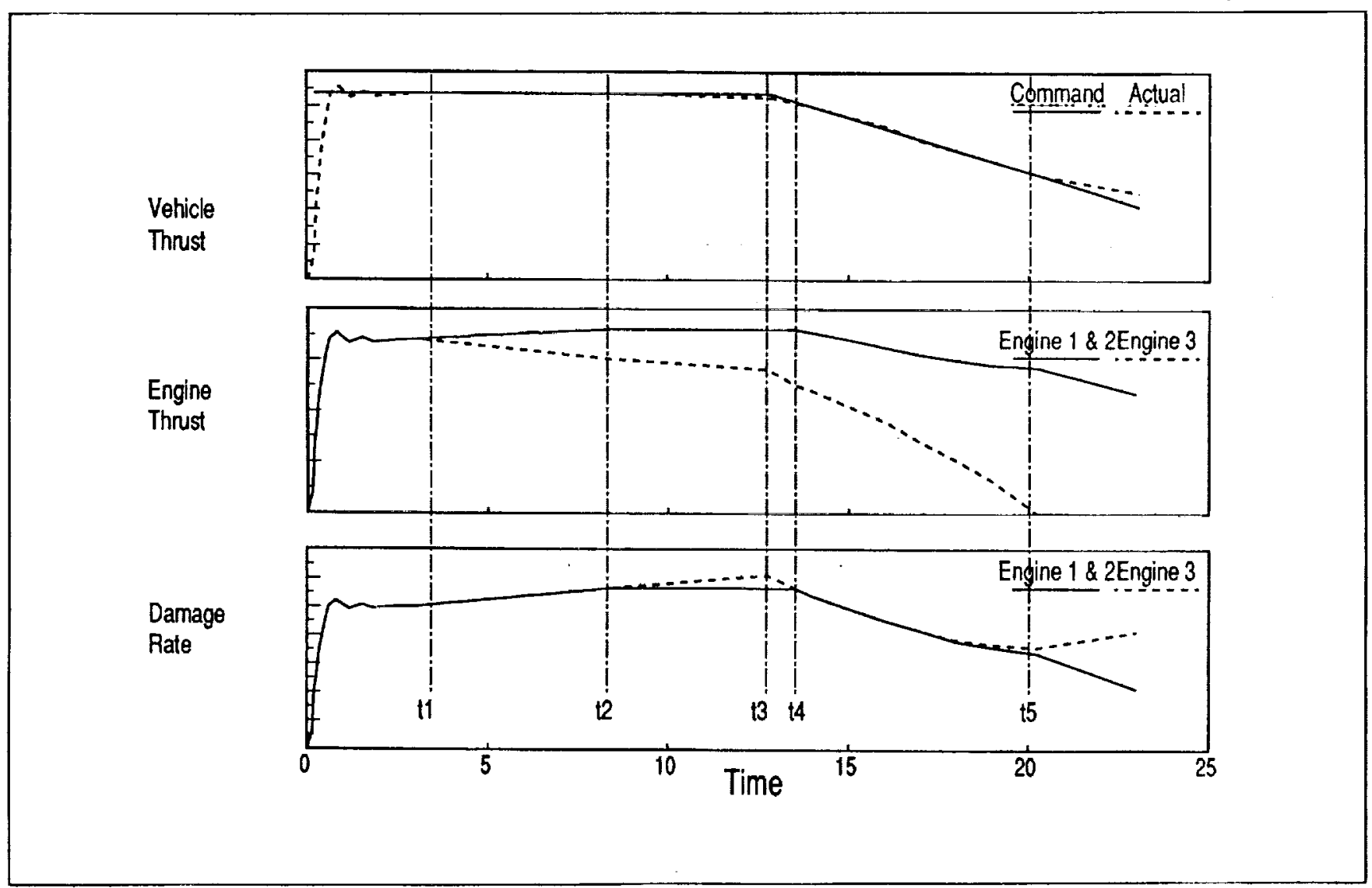

Figure 11 Results for an example Estimated Damage LEC. 


\section{FATIGUE DAMAGE EXAMPLE}

A simple hydraulic actuator example is used to illustrate an implicit form of LEC (figure 12). The assumption in this example is that the actuator rod is the life critical element and its life is the resource to be managed while obtaining system performance. System performance is defined in terms of a positloning task. The mass at the end of the rod has to be placed, altemately, at positions $X 1$ and $X 2$. The number of placements (i.e. the number of times $x=X 1$ or $X 2$ ) is $N$. Also, The time to achieve $N$ is $T$. The performance is then defined to be

$$
P=c_{N} N-c_{T} T
$$

The first term in equation (8) is related to dynamic system performance and the second term is related to accumulated damage. This performance measure can be interpreted in a number of ways and represents a fairly general measure. For example, if the positioning servomechanism represents a production assembly machine, $\mathrm{N}$ can represent the number of parts manufactured, $T$ the time to manufacture the $N$ parts, $c_{N}$ the selling price per part, and $c_{T}$ the cost per unit time to manufacture. In this case $P$ becomes a measure of profit. Another interpretation is the positioning of an control surface on an aircraft. Here $\mathbf{N}$ would represent the number of times a command is given to move the control surface, $T$ represents the time required to achieve the desired commanded position, and the constants $c_{N}$ and $c_{T}$ represent the tradeoff between positioning accuracy and time to achieve the position.

The system description for this example is chosen to be

$$
\begin{aligned}
& \dot{z}=A z+B x_{\text {tra }} \\
& x=C z \\
& F=x_{\text {tres }}-x
\end{aligned}
$$

where $z$ is the system state (position and velocity), $x$ is the position, $F$ is the Force applied to the rod, and $x_{\text {trai }}$ is the commanded position, and

$$
A=\left[\begin{array}{cc}
0 & 1 \\
-56 & -5
\end{array}\right] ; \quad B=\left[\begin{array}{c}
0 \\
50
\end{array}\right] ; \quad C=\left[\begin{array}{ll}
1 & 0
\end{array}\right]
$$

The life of the rod is a function of the compressive and tensile forces, $F$, applied to the rod. These forces are a function of $N$ (implicitly the number of stress cycles) and $T$ (actuator rod load depends on acceleration which determines $T$ ) thus

$$
D=\operatorname{Damage}(r o d)=f(F)=f(N, T)
$$

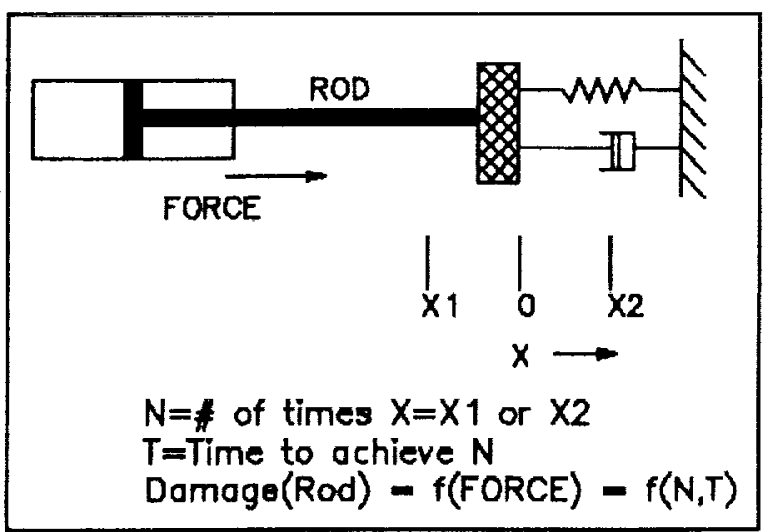

Flgure 12 A hydraulic actuator position system. 


\section{FATIGUE DAMAGE EXAMPLE RESULTS}

The example system of equations (8)-(10) was used to illustrate LEC. In figure 13 pulse sequence trajectory number 1 was applied to the system. Also shown in flgure 13 are the system position and scaled force trajectory resulting from pulse trajectory 1 . The performance endpoints were selected as $\times 1=-X_{2}=1$. In this case $\mathrm{N}=11$ and $\mathrm{T}=4.9 \mathrm{sec}$. A damage analysis was accomplished on the simulated force trajectory, using equations (1)(5). Here five major stress-strain cycles (as determined by the scaled force trajectory) were observed and used to calculate damage. In this case $D=.0213$ units of damage based upon a total component life of 1 unit were predicted.

A modified commanded pulse sequence trajectory, called trajectory number 2, was applied to the same system. The commanded trajectory, the system position, and the scaled force for case 2 is shown in figure 14. In case 2, $\mathrm{N}-9$ and $\mathrm{T}=4.6 \mathrm{sec}$. The damage analysis in this case was based upon an observed count of 4 major stress-strain cycles and 8 minor cycles. Minor cycles (e.g. $c_{1}$ in figure 14) are so named because they result in very small damage. In case 2, D=.0121. Because the commanded pulse trajectory has been slowed slightly, the resultant force trajectory has smaller peak magnitudes.

The time to failure will be $T_{f}=230 \mathrm{sec}$. for case 1 and $T_{f}=380 \mathrm{sec}$. for case 2 . The number of endpoint placements at $T_{\text {, is }} \mathrm{N}_{1}=516$ for Consequently, the stress-strain cycles have smaller magnitudes and the damage will be less.case 1 and $N_{2}-743$ for case 2. Given the constants of performance of equation (7) are $C_{N}=2$ and $C_{T}=1$, the resultant performance for case 1 is $P_{1}=802$. For case $2, P_{2}=1106$. These performance constants can be interpreted as described above. Case 2 offers better total system performance, as defined by equation (7), by adopting a strategy that obtains endpoint placements at a slightly longer time and results in a much more effective use of the lifetime of the critical component. Assuming that the system will be operated to failure with the two pulse sequences selected, the example results are summarized in Table III.

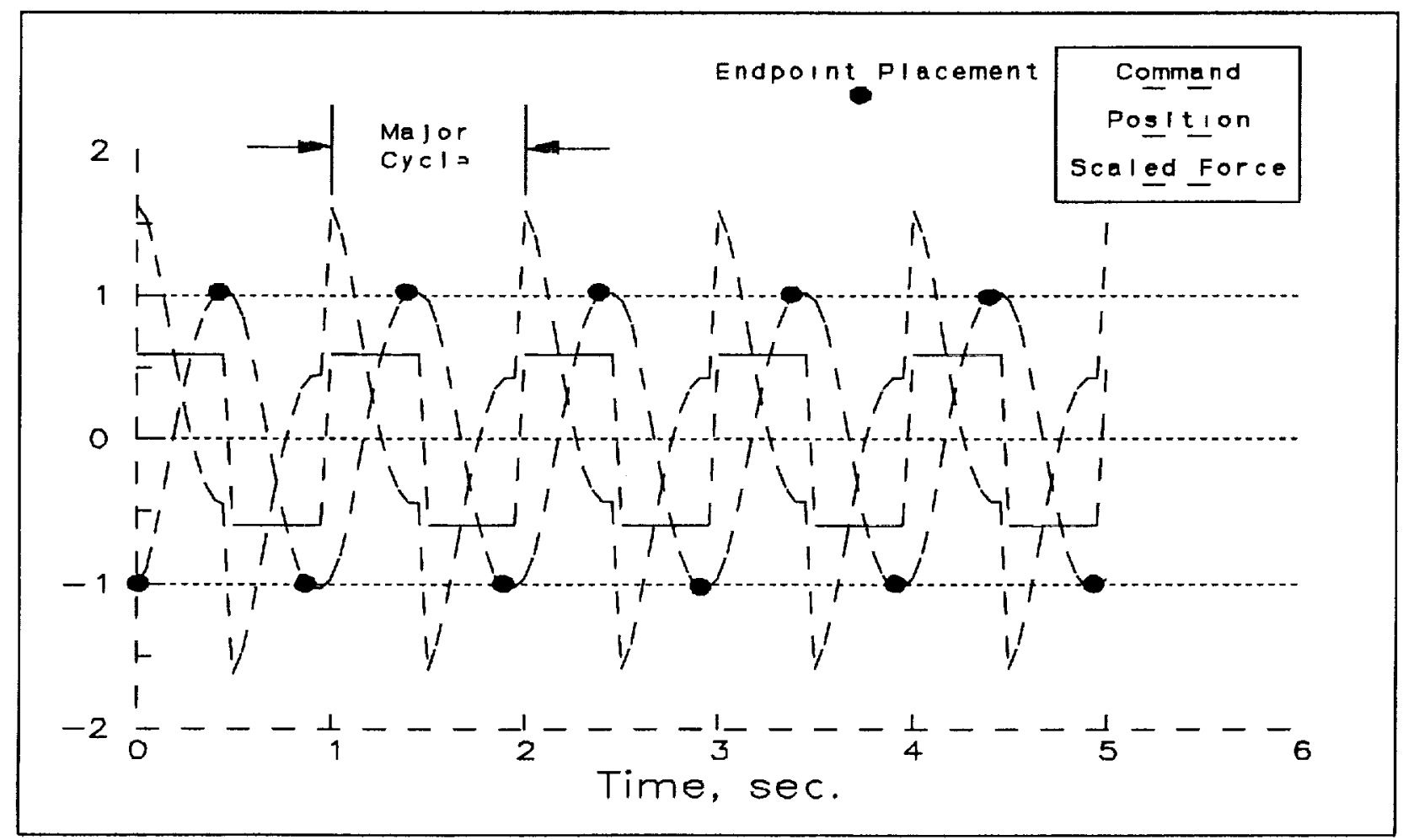

Figure 13 Example results for Case 1 trajectory command. 


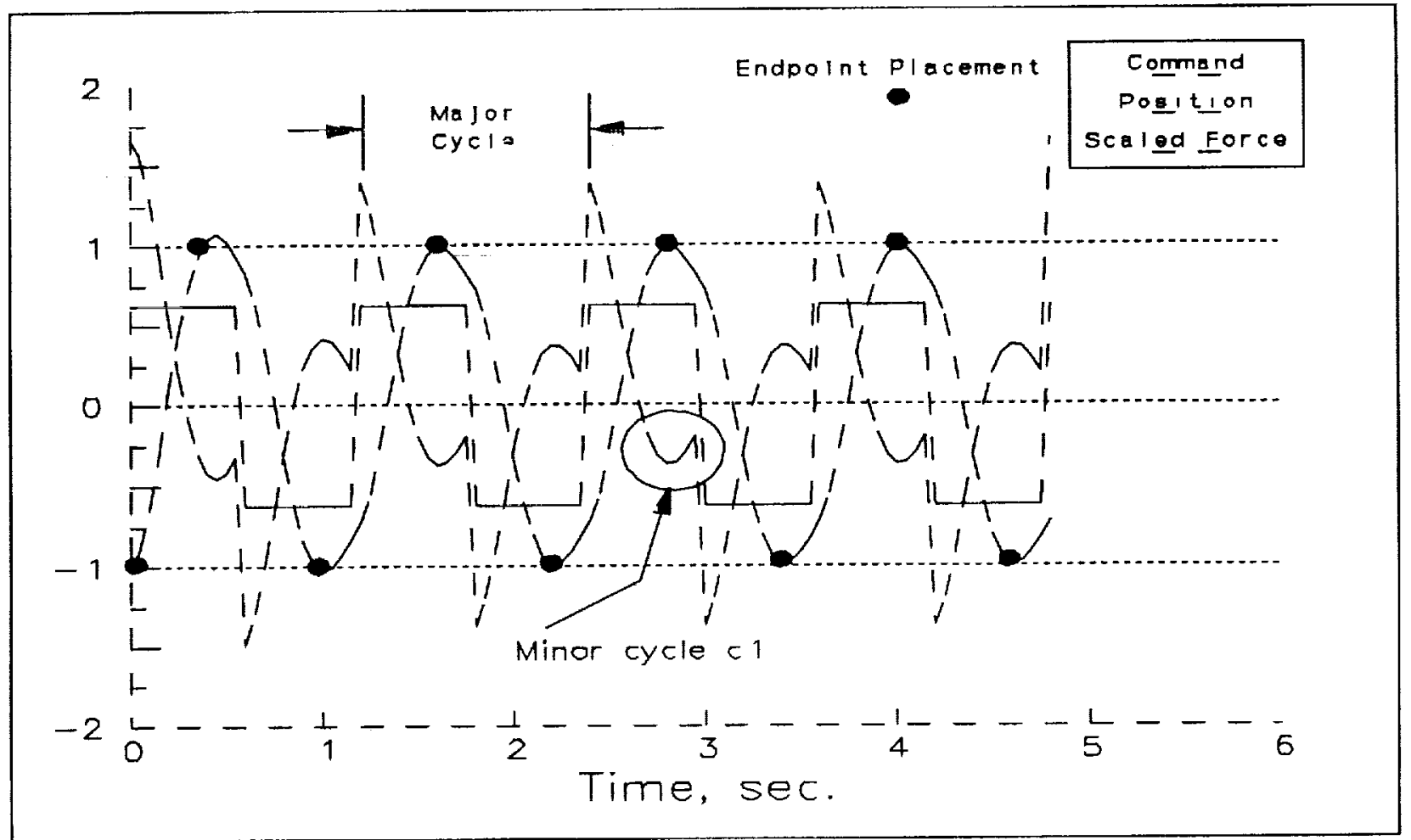

Flgure 14 Example results for Case 2 trajectory command.

Table III EXAMPLE LIFE EXTENDING CONTROL RESULTS

$\begin{array}{llllllllll}\text { CASE } & \mathrm{N} & \mathrm{T}, \mathrm{sec} & \mathrm{N} / \mathrm{T} & \mathrm{F}_{\mathrm{MAX}} & \text { CYCLES } & \mathrm{D} & \mathrm{T}_{\mathrm{f}} & \mathrm{N}_{\text {tool }} & \text { PERFORMANCE } \\ 1 & 11 & 4.9 & 2.2448 & 1.6 & \text { 5 MAJOR } & 0.0213230 & 516 & 802 \\ 2 & 9 & 4.6 & 1.9565 & 1.4 & \begin{array}{l}\text { 4 MAJOR } \\ \text { 8 } 0.0121380\end{array} & 743 & 1106 \\ & & & & & \text { 8 MINOR } & & & \end{array}$

\section{CONCLUSIONS}

The concept of Life Extending Control was introduced. The procedure for the prediction of the mechanical fatigue life of critical system components using a local, cyclic stress-strain approach was described. Possible extensions to this cyclic damage prediction approach were presented based on the identification of a model from elementary forms. Several candidate elementary forms were presented. These extensions would result in a continuous or differential form of the damage prediction model.

Two possible approaches to Life Extending Control based on the existing cyclic damage prediction method, called implicit LEC and life management LEC approach were proposed. The implicit approach uses an off-line optimization procedure to find potentially life extending controls. The life management approach uses a hierarchical structure to accomplish a performance/ife tradeoff and to select life extending commanded trajectories. Two possible approaches to Life Extending Control based on the proposed continuous damage prediction method, called measured variables LEC and estimated variables LEC approach were defined. Here damage 
measurements or estimates would be used directly in the LEC. One implementation would be a life setpoint approach using nonlinear feedback control methods to regulate incremental damage. An example of the estimated damage LEC based on an empirical damage estimate was presented. A simple hydraulic actuator driven, position control system example is used to illustrate the main ideas behind Life Extending Control based on a current cyclic fatigue damage model. Results from this example demonstrate that overall system performance, that is, dynamic plus life, can be maximized by accounting for critical component damage in the control design.

\section{ACKNOWLEDGEMENT}

The authors would like to thank Mr. Ed Nemeth and Mr. Ron Anderson of Rocketdyne for generating the results of Figure 11.

\section{REFERENCES}

1. Merrill, Walter C. and Lorenzo, Carl F.: A Reusable Rocket Engine Intelligent Control. NASA TM 100963, AIAA-88-3114, July 11-13, 1988. Presented at the 1988 Joint Propulsion Conference, Boston, MA.

2. Lorenzo, Carl F. and Merrill, Walter C.: An Intelligent Control System for Rocket Engines: Need, Vision, and Issues. May 23-25, 1990, Paper WE 7:15 1990 American Control Conference, San Diego, CA.

3. Dowling, N. E., Brose, W. R., and Wilson, W. K.; Notched Member Fatigue Life Predictions by the Local Strain Approach, in Fatigue Under Complex Loading: Analyses and Experiments, Librany of Congress CCN 77-85454, Society of Automotive Engineers, Inc. 1977

4. Saltsman, J. F. and Halford, G. R.: Life Prediction of Thermomechanical Fatigue Using A Total Strain Version of Strainrange Partitioning (SRP) - A Proposal. NASA TP-2779, February 1988.

5. Troudet, T. and Merrill, W.: A Real Time Neural Net Estimator of Fatigue Life. Proceedings of the Int. Joint Conf. on Neural Networks, June 1990.

6. Saridis, G. N., and Valavanis, K. P.; "Analytical Design of Intelligent Machines," Automatica, VI. 24, No. 2, pp. 123-133, 1988

7. Nemeth, Ed and Merrill, Walter C.: A Framework for Intelligent Control of Reusable Rocket Engines. Advanced Earth-to-Orbit Propulsion Technology 1990, NASA CP 3092, Vol I, pp 510-523, May 1990. 


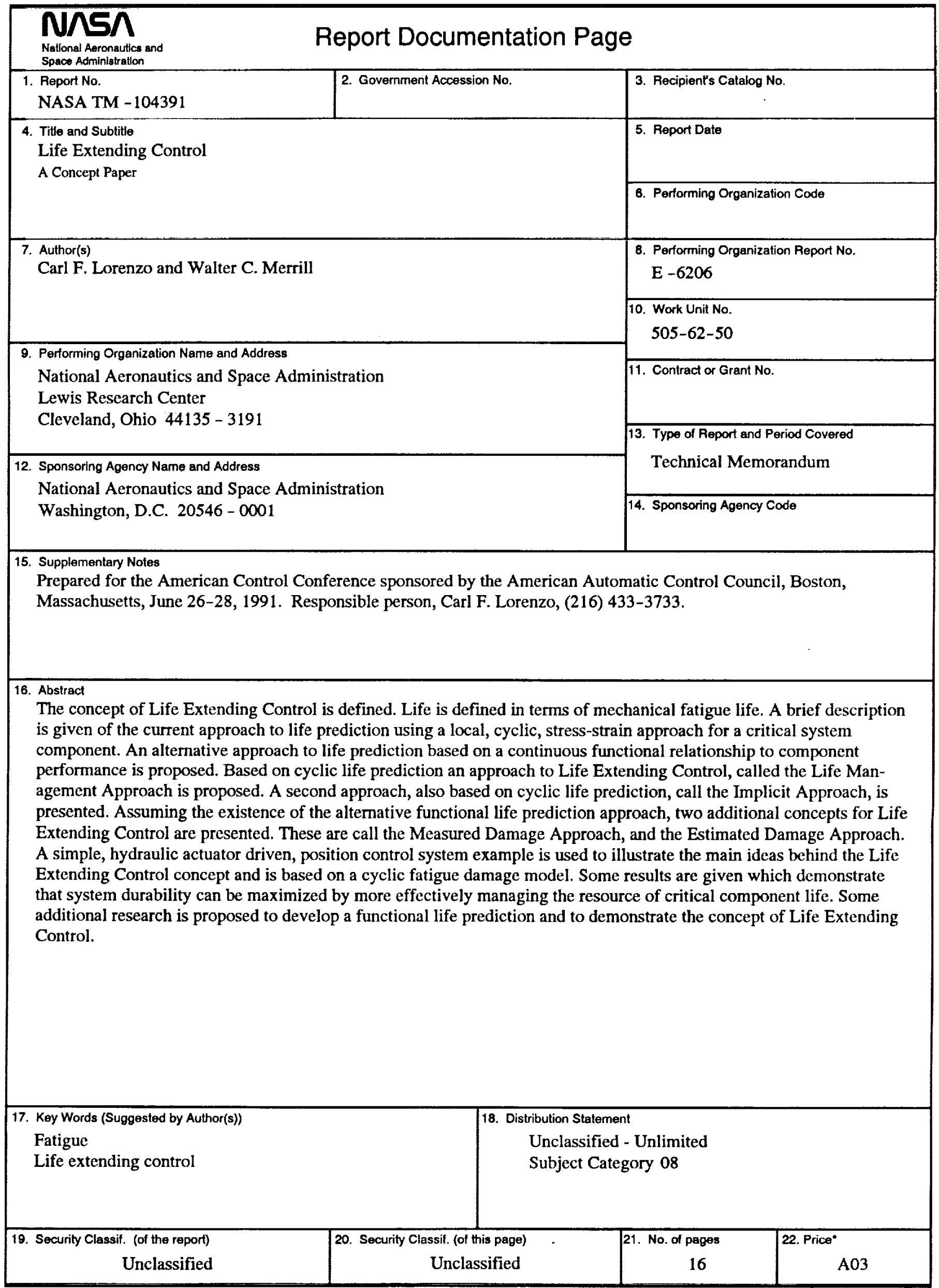

